PROCEEDINGS OF THE

AMERICAN MATHEMATICAL SOCIETY

Volume 134, Number 6, Pages 1783-1789

S 0002-9939(05)08139-6

Article electronically published on December 15, 2005

\title{
ON THE REFLEXIVITY OF MULTIVARIABLE ISOMETRIES
}

\author{
JÖRG ESCHMEIER
}

(Communicated by Joseph A. Ball)

\begin{abstract}
Let $A \subset C(K)$ be a unital closed subalgebra of the algebra of all continuous functions on a compact set $K$ in $\mathbb{C}^{n}$. We define the notion of an $A$-isometry and show that, under a suitable regularity condition needed to apply Aleksandrov's work on the inner function problem, every $A$-isometry $T \in L(\mathcal{H})^{n}$ is reflexive. This result applies to commuting isometries, spherical isometries, and more generally, to all subnormal tuples with normal spectrum contained in the Bergman-Shilov boundary of a strictly pseudoconvex or bounded symmetric domain.
\end{abstract}

\section{INTRODUCTION}

Let $\mathcal{S} \subset L(\mathcal{H})$ be an arbitrary family of bounded linear operators on a complex Hilbert space $\mathcal{H}$. We denote by $\operatorname{Lat}(\mathcal{S})$ the set of all closed linear subspaces of $\mathcal{H}$ that are invariant under every operator $S \in \mathcal{S}$. The set

$$
\operatorname{Alg} \operatorname{Lat}(\mathcal{S})=\{C \in L(\mathcal{H}) ; \operatorname{Lat}(C) \supset \operatorname{Lat}(\mathcal{S})\}
$$

is a subalgebra of $L(\mathcal{H})$ which contains $\mathcal{S}$ and is closed in the weak operator topology (WOT). The family $\mathcal{S}$ is called reflexive if

$$
\operatorname{Alg} \operatorname{Lat}(\mathcal{S})=\mathcal{W}_{\mathcal{S}}
$$

where the right-hand side denotes the smallest WOT-closed subalgebra of $L(\mathcal{H})$ containing $\mathcal{S}$ and the identity operator $1_{\mathcal{H}}$.

Sarason [17] proved in 1966 that analytic Toeplitz operators on the Hardy space $H^{2}(\mathbb{D})$ over the unit disc $\mathbb{D}$ and commuting families of normal operators are reflexive. In 1971 it was shown by Deddens [8] that all isometries are reflexive. These results were generalized in a paper of Olin and Thomson [15] from 1980 which showed that every subnormal operator is reflexive. Whether the corresponding result holds for subnormal tuples $T=\left(T_{1}, \ldots, T_{n}\right) \in L(\mathcal{H})^{n}$ of Hilbert space operators, is one of the major open problems in this area of multivariable invariant subspace theory.

The result of Deddens on the reflexivity of single isometries was extended by $\mathrm{Li}$ and McCarthy [13] to families of commuting isometries on separable Hilbert spaces and by Bercovici [4] to arbitrary families of commuting isometries. In a recent paper of Didas [10] the result of Bercovici is used to show that every spherical isometry,

Received by the editors January 14, 2005 and, in revised form, January 31, 2005.

2000 Mathematics Subject Classification. Primary 47A15; Secondary 47A13, 47B20, 47L45. 
that is, each commuting system $T=\left(T_{1}, \ldots, T_{n}\right) \in L(\mathcal{H})^{n}$ with

$$
\sum_{i=1}^{n} T_{i}^{*} T_{i}=1_{\mathcal{H}}
$$

is reflexive.

An $n$-tuple $T=\left(T_{1}, \ldots, T_{n}\right) \in L(\mathcal{H})^{n}$ consists of commuting isometries if and only if $T$ is subnormal and the joint spectrum of its minimal normal extension is contained in the Shilov boundary of the polydisc algebra $A\left(\mathbb{D}^{n}\right)$. Analogously, the class of spherical isometries consists precisely of those subnormal $n$-tuples $T=$ $\left(T_{1}, \ldots, T_{n}\right)$ with the property that the joint spectrum of their minimal normal extension is contained in the Shilov boundary of the ball algebra $A\left(\mathbb{B}_{n}\right)$. In this note we replace the polydisc and ball algebra by suitable closed subalgebras $A \subset C(K)$ over compact sets $K$ in $\mathbb{C}^{n}$ to define the notion of an $A$-isometry and prove that, under a regularity condition needed to apply Aleksandrov's work [1] on the existence of inner functions, every $A$-isometry $T \in L(\mathcal{H})^{n}$ is reflexive.

The main tools are the above-cited result of Bercovici [4] on the reflexivity of commuting families of isometries and the work of Aleksandrov [1] on the existence of inner functions for uniform algebras.

\section{Reflexivity of $A$-isometries}

Recall that a commuting tuple $T=\left(T_{1}, \ldots, T_{n}\right) \in L(\mathcal{H})^{n}$ on a complex Hilbert space $\mathcal{H}$ is called subnormal if it can be extended to a commuting tuple $N=$ $\left(N_{1}, \ldots, N_{n}\right) \in L(\mathcal{K})^{n}$ of normal operators on a larger Hilbert space $\mathcal{K} \supset \mathcal{H}$. A normal extension $N$ of $T$ as above is called minimal if $\mathcal{K}$ is the only reducing subspace for $N$ that contains $\mathcal{H}$. All minimal normal extensions of a subnormal tuple $T$ are unitarily equivalent and, for every normal extension $N$ of $T$, there is a reducing subspace $\mathcal{K}_{0}$ for $N$ which contains $\mathcal{H}$ such that $N \mid \mathcal{K}_{0}$ is a minimal normal extension of $T$.

Let $T \in L(\mathcal{H})^{n}$ be subnormal with minimal normal extension $N \in L(\mathcal{K})^{n}$. The normal spectrum of $T$ defined as $\sigma_{n}(T)=\sigma(N)$, where $\sigma(N)$ denotes the Taylor spectrum of $N$, is independent of the choice of $N$. A result of Putinar [16] shows that $\sigma_{n}(T) \subset \sigma(T)$. Let $\mu$ be a scalar spectral measure of $N$. Denote by $\Psi: L^{\infty}(\mu) \rightarrow L(\mathcal{H})$ the $L^{\infty}$-functional calculus of the normal tuple $N$. The restriction algebra

$$
\mathcal{R}(T)=\left\{f \in L^{\infty}(\mu) ; \Psi(f) \mathcal{H} \subset \mathcal{H}\right\}
$$

is a weak* closed subalgebra of $L^{\infty}(\mu)$, and the induced algebra homomorphism

$$
\gamma_{T}: \mathcal{R}(T) \rightarrow L(\mathcal{H}), \quad f \mapsto \Psi(f) \mid \mathcal{H}
$$

is isometric and weak ${ }^{*}$ continuous if $L(\mathcal{H})$ is equipped with its weak ${ }^{*}$ topology as the dual space of the trace class operators (see Proposition 1.1 in 7 ). Since all scalar spectral measures of $T$, that is, measures $\mu$ arising as a scalar spectral measure of some minimal normal extension $N$ of $T$, are mutually absolutely continuous, both the restriction algebra $\mathcal{R}(T)$ and the algebra homomorphism $\gamma_{T}$ are independent of the choice of $N$.

Let $K \subset \mathbb{C}^{n}$ be a compact subset, and let $A \subset C(K)$ be a unital closed subalgebra containing the restrictions of the polynomials. We write $S(A)$ for the Shilov boundary of $A$, that is, the smallest closed set $S \subset K$ such that

$$
\|f\|_{\infty, K}=\|f\|_{\infty, S} \quad(f \in A) .
$$


Denote by $M^{+}(K)$ the set of all positive regular Borel measures on $K$. For $\mu \in$ $M^{+}(K)$, the triple $(A, K, \mu)$ is called regular in the sense of Aleksandrov [1] if, for each function $\varphi \in C(K)$ with $\varphi>0$, there exists a sequence $\left(f_{k}\right)$ in $A$ with $\left|f_{k}\right|<\varphi$ for all $k$ and $\lim _{k \rightarrow \infty}\left|f_{k}\right|=\varphi \mu$-almost everywhere. If the triple $(A, K, \mu)$ is regular, then the support of $\mu$ is necessarily contained in $S(A)$. For this and other properties of regular triples, the reader is referred to [1, [2].

Fix a unital closed subalgebra $A \subset C(K)$ containing the polynomials. A subnormal tuple $T \in L(\mathcal{H})^{n}$ will be called $A$-subnormal if $\sigma_{n}(T) \subset K$ and $A \subset \mathcal{R}(T)$. By an $A$-isometry we mean an $A$-subnormal tuple with $\sigma_{n}(T) \subset S(A)$. An $A$-isometry $T \in(\mathcal{H})^{n}$ is said to be regular if the triple $(A, K, \mu)$ is regular for some, or equivalently, every scalar spectral measure $\mu$ of $T$. Here $\mu$ is regarded as a measure on $K$ via trivial extension.

With these notations our main result can be formulated as follows.

Theorem 1. Let $A \subset C(K)$ be a unital closed subalgebra over some compact set $K$ in $\mathbb{C}^{n}$ containing the polynomials. Then every regular A-isometry is reflexive.

In the following we fix $A$ and $K$ as in Theorem 1. For any given measure $\mu \in M^{+}(K)$, we shall denote by $H_{A}^{\infty}(\mu)$ the weak* closure of $A$ in $L^{\infty}(\mu)$ and by $H_{A}^{2}(\mu)$ the norm-closure of $A$ in $L^{2}(\mu)$.

The proof of Theorem 1 will be based on the result of Bercovici [4 cited above and on the following observation of Aleksandrov (Corollary 29 in 11) on the existence of $\mu$-inner functions.

Proposition 2 (Aleksandrov). Let $\mu \in M^{+}(K)$ be a measure with $\mu(\{z\})=0$ for every $z \in K$ such that $(A, K, \mu)$ is regular. Then the weak $k^{*}$ closure of the set $\left\{f \in H_{A}^{\infty}(\mu) ;|f|=1 \mu\right.$-almost everywhere $\}$ contains the closed unit ball of $A$.

As usual we call a measure $\mu \in M^{+}(K)$ continuous if $\mu(\{z\})=0$ for every $z \in K$ and discrete if there is a countable set $D \subset K$ with $\mu(K \backslash D)=0$. For an arbitrary measure $\mu \in M^{+}(K)$, the set $D=\{z \in K ; \mu(\{z\})>0\}$ is countable, and the measures $\mu_{c}, \mu_{d} \in M^{+}(K)$ defined by

$$
\mu_{c}(A)=\mu(A \cap(K \backslash D)), \quad \mu_{d}(A)=\mu(A \cap D)
$$

yield the unique representation of $\mu$ as a sum $\mu=\mu_{c}+\mu_{d}$ of a continuous measure $\mu_{c}$ and a discrete measure $\mu_{d}$. The following observation of Aleksandrov (Proposition 15 in [1]) allows us to reduce the proof of Theorem 1 to the case of $A$-isometries with continuous scalar spectral measure.

Lemma 3. Suppose that the triple $(A, K, \mu)$ is regular. Then the orthogonal decomposition $H_{A}^{2}(\mu)=H_{A}^{2}\left(\mu_{c}\right) \oplus L^{2}\left(\mu_{d}\right)$ holds.

Proof. Since this result is stated without proof in 1, we indicate the main ideas.

For $\mu \in M^{+}(K)$, define $\mu_{c}$ and $\mu_{d}$ as the continuous and discrete part of $\mu$ as in the section leading to the lemma. Denote by $\varkappa_{c}$ and $\varkappa_{d}$ the characteristic functions of $K \backslash D$ and $D$, respectively. Then the map

$$
\sigma: L^{2}\left(\mu_{c}\right) \oplus L^{2}\left(\mu_{d}\right) \rightarrow L^{2}(\mu), \quad(f, g) \mapsto f \varkappa_{c}+g \varkappa_{d}
$$

is easily seen to be a unitary operator between Hilbert spaces with inverse given by $\rho: L^{2}(\mu) \rightarrow L^{2}\left(\mu_{c}\right) \oplus L^{2}\left(\mu_{d}\right)$,

$$
\rho\left([f]_{L^{2}(\mu)}\right)=\left([f]_{L^{2}\left(\mu_{c}\right)},[f]_{L^{2}\left(\mu_{d}\right)}\right) .
$$


To prove the equality $\rho\left(H_{A}^{2}(\mu)\right)=H_{A}^{2}\left(\mu_{c}\right) \oplus L^{2}\left(\mu_{d}\right)$, it suffices to show the inclusion $\sigma\left(L^{2}\left(\mu_{d}\right)\right) \subset H_{A}^{2}(\mu)$. Since $\mu_{d}$ is discrete, it is enough to prove that the characteristic function $\varkappa_{\{w\}}$ of every point $w \in D$ belongs to $H_{A}^{2}(\mu)$. Using the density of $C(K)$ in $L^{2}(\mu)$, one obtains a sequence $\left(f_{k}\right)$ in $C(K)$ converging to $\varkappa_{\{w\}}$ in $L^{2}(\mu)$ such that $0<f_{k}<2$ for all $k$. The hypothesis that $(A, K, \mu)$ is regular allows us to choose (Theorem 37 in [1]) functions $g_{k} \in A$ with $\left|g_{k}\right| \leq f_{k}$ and

$$
\mu\left(\left\{\left|g_{k}\right| \neq f_{k}\right\}\right)<\mu(\{w\}) /(k+1) \quad(k \in \mathbb{N}) .
$$

Obviously, one can achieve that $g_{k}(w)=f_{k}(w)$ holds for all $k$. In view of the estimate

$$
\begin{aligned}
& \left\|g_{k}-\varkappa_{\{w\}}\right\|_{L^{2}(\mu)}^{2}=\left|g_{k}(w)-1\right|^{2} \mu(\{w\})+\int_{K \backslash\{w\}}\left|g_{k}\right|^{2} d \mu \\
& \leq\left|f_{k}(w)-1\right|^{2} \mu(\{w\})+\left\|f_{k}-\varkappa_{\{w\}}\right\|_{L^{2}(\mu)}^{2}+4 \mu\left(\left\{\left|g_{k}\right| \neq f_{k}\right\}\right)
\end{aligned}
$$

it is clear that $\left(g_{k}\right)$ converges to $\varkappa_{\{w\}}$ in $L^{2}(\mu)$.

Each subnormal tuple $T \in L(\mathcal{H})^{n}$ admits a unique orthogonal decomposition

$$
T=T_{0} \oplus T_{1} \in L\left(\mathcal{H}_{0} \oplus \mathcal{H}_{1}\right)^{n}
$$

such that $T_{1} \in L\left(\mathcal{H}_{1}\right)^{n}$ is normal and $T_{0} \in L\left(\mathcal{H}_{0}\right)^{n}$ is pure, that is, possesses no non-zero reducing subspace $M$ for which $T_{0} \mid M$ is normal.

Lemma 4. Let $T=T_{0} \oplus T_{1} \in L\left(\mathcal{H}_{0} \oplus \mathcal{H}_{1}\right)^{n}$ be the decomposition of a given subnormal tuple $T \in L(\mathcal{H})^{n}$ into its pure part $T_{0} \in L\left(\mathcal{H}_{0}\right)^{n}$ and normal part $T_{1} \in$ $L\left(\mathcal{H}_{1}\right)^{n}$. If $T$ is a regular $A$-isometry, then so is $T_{0}$.

Proof. Let $N \in L(\mathcal{K})^{n}$ be a minimal normal extension of $T$. Denote by $E$ the projection-valued spectral measure of $N$. The space

$$
\mathcal{K}_{0}=\bigvee\left(N^{* k} \mathcal{H}_{0} ; k \in \mathbb{N}^{n}\right)
$$

reduces $N$, and $N_{0}=N \mid \mathcal{K}_{0}$ is a minimal normal extension of $T_{0}$. Hence $\sigma_{n}\left(T_{0}\right)=$ $\sigma\left(N_{0}\right) \subset \sigma(N) \subset S(A)$.

Set $\mathcal{K}_{1}=\mathcal{K} \ominus \mathcal{K}_{0}$. Choose separating vectors $x_{0} \in \mathcal{K}_{0}$ for $N_{0}$ and $x_{1} \in \mathcal{K}_{1}$ for $N_{1}=N \mid \mathcal{K}_{1}$. Since the von Neumann algebras generated by $N, N_{0}$ and $N_{1}$ satisfy the relation

$$
W^{*}(N) \subset W^{*}\left(N_{0}\right) \oplus W^{*}\left(N_{1}\right),
$$

the vector $x=x_{0}+x_{1}$ is a separating vector for $N$. Because of

$$
\langle E(\cdot) x, x\rangle=\left\langle E(\cdot) x_{0}, x_{0}\right\rangle+\left\langle E(\cdot) x_{1}, x_{1}\right\rangle
$$

the scalar spectral measure $\mu_{0}=\left\langle E(\cdot) x_{0}, x_{0}\right\rangle$ of $N_{0}$ is absolutely continuous with respect to the scalar spectral measure $\mu=\langle E(\cdot) x, x\rangle$ of $N$. Hence $\left(A, K, \mu_{0}\right)$ remains regular.

The projection-valued spectral measure $E_{0}$ of $N_{0}$ acts as

$$
E_{0}(A)=E(A) \mid \mathcal{K}_{0} \in L\left(\mathcal{K}_{0}\right),
$$

where $A$ runs through all Borel subsets of $\sigma\left(N_{0}\right)$. Hence the $L^{\infty}$-functional calculi of $N_{0}$ and $N$ are related by

$$
\Psi_{N_{0}}(f)=\Psi(f) \mid \mathcal{K}_{0} \quad\left(f \in L^{\infty}(\mu)\right) .
$$


It follows that $\Psi_{N_{0}}(f) \mathcal{H}_{0} \subset \mathcal{H}$ for every function $f \in A$. Since $\mathcal{H}_{1}$ is a reducing subspace for $N$, we find that

$$
\left\langle\Psi_{N_{0}}(f) x, y\right\rangle=\left\langle x, \Psi(f)^{*} y\right\rangle=0
$$

for all $f \in A$ and $x \in \mathcal{H}_{0}, y \in \mathcal{H}_{1}$. Therefore $A \subset \mathcal{R}\left(T_{0}\right)$, and the proof is complete.

Let $\mathcal{A} \subset L(\mathcal{H})$ be a weak* closed subalgebra. The set of all weak* continuous linear functionals on $\mathcal{A}$ can be identified isometrically with the quotient space $Q_{\mathcal{A}}=$ $\mathcal{C}^{1}(\mathcal{H}) /^{\perp} \mathcal{A}$, where $\mathcal{C}^{1}(\mathcal{H})$ denotes the Banach space of all trace class operators on $\mathcal{H}$. For $x, y \in \mathcal{H}$, we write $x \otimes y \in Q_{\mathcal{A}}$ for the equivalence class of the rank-one operator $\mathcal{H} \rightarrow \mathcal{H}, \xi \mapsto\langle\xi, y\rangle$. The associated weak* continuous functional on $\mathcal{A}$ acts as $x \otimes y(A)=\langle A x, y\rangle$.

Recall that $\mathcal{A}$ is said to possess the factorisation property $\left(\mathbb{A}_{1}\right)$ if every element $L \in Q_{\mathcal{A}}$ is of the form $L=x \otimes y$ with suitable vectors $x, y \in \mathcal{H}$.

To reduce Theorem 1 to the pure case, we shall use the following well-known result (cf. for instance Lemma 4.4.1 in [9]).

Lemma 5. Suppose that $\mathcal{A}_{i} \subset L\left(\mathcal{H}_{i}\right)(i=1,2)$ are reflexive subalgebras with

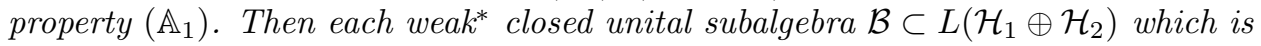
contained in $\mathcal{A}_{1} \oplus \mathcal{A}_{2}$ is reflexive and has property $\left(\mathbb{A}_{1}\right)$. Furthermore, the weak and weak operator topology coincide on $\mathcal{B}$.

Now we have gathered all the tools necessary for the proof of our main result.

Proof of Theorem 1. Let $T \in L(\mathcal{H})^{n}$ be a regular $A$-isometry. Denote by $T=$ $T_{0} \oplus T_{1} \in L\left(\mathcal{H}_{0} \oplus \mathcal{H}_{1}\right)^{n}$ the decomposition of $T$ into its pure part $T_{0} \in L\left(\mathcal{H}_{0}\right)^{n}$ and normal part $T_{1} \in L\left(\mathcal{H}_{1}\right)^{n}$. Let us observe first that it suffices to show that $T_{0}$ is reflexive and that $\operatorname{Alg} \operatorname{Lat}\left(T_{0}\right)=\mathcal{W}_{T_{0}}$ has property $\left(\mathbb{A}_{1}\right)$. Indeed, since the normal part $T_{1}$ satisfies both of these properties ([17] and Proposition 2.05 in [5]) and since the weak* closed subalgebra $\mathcal{A}_{T}$ of $L(\mathcal{H})$ generated by $T$ is contained in $\mathcal{W}_{T_{0}} \oplus \mathcal{W}_{T_{1}}$, an application of Lemma 5 will complete the proof of Theorem 1.

Since by Lemma 4 the pure part $T_{0}$ is a regular $A$-isometry again, it suffices to assume from the very beginning that $T$ is pure and to show that $T$ is reflexive and that $\operatorname{AlgLat}(\mathrm{T})=\mathcal{W}_{\mathrm{T}}$ has property $\left(\mathbb{A}_{1}\right)$. Thus let us fix a pure regular $A$-isometry $T \in L(\mathcal{H})^{n}$. Choose a minimal normal extension $N \in L(\mathcal{K})^{n}$ of $T$ and denote by $E$ its projection-valued spectral measure. Exactly as in the one-variable case (Proposition V.17.14 in [6]) one can show that there is a separating vector $x \in \mathcal{H}$ for $N$. Let $\mu=\langle E(\cdot) x, x\rangle$ be the associated scalar spectral measure of $T$, and let $\Psi: L^{\infty}(\mu) \rightarrow L(\mathcal{K})$ be the $L^{\infty}$-functional calculus of $N$. Since

$$
\|\Psi(f) x\|^{2}=\left\langle\Psi\left(|f|^{2}\right) x, x\right\rangle=\|f\|_{L^{2}(\mu)}^{2}
$$

for all $f \in L^{\infty}(\mu)$, the map $A \rightarrow \mathcal{H}, f \mapsto \Psi(f) x$, extends to a unique isometry $j: H_{A}^{2}(\mu) \rightarrow \mathcal{H}$. The relation

$$
j(g f)=\Psi(g f) x=\Psi(g) j(f)
$$

obviously holds for all functions $f, g \in A$. A continuity argument implies that $j(g f)=\Psi(g) j(f)$ holds for all $g \in H_{A}^{\infty}(\mu)$ and $f \in H_{A}^{2}(\mu)$. In particular, we find that

$$
j \circ M_{z_{i}}=T_{i} \circ j \quad(1 \leq i \leq n),
$$


where $M_{z_{i}}: H_{A}^{2}(\mu) \rightarrow H_{A}^{2}(\mu), f \mapsto z_{i} f$, is the operator of multiplication with the $i$-th coordinate function.

Using the decomposition $H_{A}^{2}(\mu)=H_{A}^{2}\left(\mu_{c}\right) \oplus L^{2}\left(\mu_{d}\right)$ from Lemma 3, we deduce that $T \mid j\left(L^{2}\left(\mu_{d}\right)\right)$ is unitarily equivalent to the normal tuple $M_{z} \mid L^{2}\left(\mu_{d}\right)$ via $j$. Our hypothesis that $T$ is pure therefore implies that $\mu=\mu_{c}$ is continuous.

Let $p \in \mathbb{C}[z]$ be a polynomial with $\|p\|_{\infty, K} \leq 1$. By Proposition 2 there is a net $\left(\theta_{i}\right)_{i \in I}$ in

$$
I=\left\{f \in H_{A}^{\infty}(\mu) ;|f|=1 \quad \mu \text {-almost everywhere }\right\}
$$

such that $p$ is the weak* limit of the net $\left(\theta_{i}\right)_{i \in I}$ in $H_{A}^{\infty}(\mu)$. It follows that $p(T)$ is the weak* limit of the net $\left(\gamma_{T}\left(\theta_{i}\right)\right)_{i \in I}$ in $L(\mathcal{H})$. Thus we have shown that

$$
\operatorname{Alg} \operatorname{Lat}(T) \subset \operatorname{Alg} \operatorname{Lat}\left(\gamma_{T}(I)\right) .
$$

Since $\gamma_{T}(I)$ consists of commuting isometries, the cited result of Bercovici (Theorem 2.3 in [4] implies that the operator algebra on the right-hand side has property $\left(\mathbb{A}_{1}\right)$. But then Proposition 2.5 in [12] shows that $T$ is reflexive and that $\operatorname{Alg} \operatorname{Lat}(T)=\mathcal{W}_{T}$ has property $\left(\mathbb{A}_{1}\right)$. Thus the proof of Theorem 1 is complete.

As a consequence of the above proof we obtain the following slightly stronger result.

Corollary 6. Let $T \in L(\mathcal{H})^{n}$ be a regular A-isometry. Then the weak* and weak operator topology coincide on the operator algebra

$$
\operatorname{Alg} \operatorname{Lat}(T)=\mathcal{W}_{T}=\mathcal{A}_{T}
$$

Furthermore, this algebra has property $\left(\mathbb{A}_{1}\right)$ and is super-reflexive, that is, each of its unital weak* ${ }^{*}$ closed subalgebras is reflexive.

Proof. The super-reflexivity of $\mathcal{A}_{T}$ is a consequence of Proposition 2.5 in [12]. The remaining assertions follow from the above proof of Theorem 1 and Lemma 5.

By specializing to the cyclic case we obtain the following result.

Corollary 7. Suppose that $\mu \in M^{+}(K)$ is a measure such that $(A, K, \mu)$ is a regular triple. Then the tuple $M_{z}=\left(M_{z_{1}}, \ldots, M_{z_{n}}\right) \in L\left(H_{A}^{2}(\mu)\right)^{n}$ consisting of the multiplication operators with the coordinate functions is reflexive, and all the remaining assertions of Corollary 6 hold.

Proof. Let $T=M_{z} \in L\left(H_{A}^{2}(\mu)\right)^{n}$. By Stone-Weierstraß the minimal normal extension of $T$ is the corresponding multiplication tuple $M_{z} \in L\left(L^{2}(\mu)\right)^{n}$. Hence $\mu$ is a scalar spectral measure for $T$, and $T$ is a regular $A$-isometry.

Let $D \subset \mathbb{C}^{n}$ be a bounded open set. Define $K=\bar{D}$ and $A=A(D)$ as the Banach algebra of all continuous functions on $\bar{D}$ that are analytic on $D$. If $D=\mathbb{B}_{n}$ is the open Euclidean unit ball, then $S(A)=\partial \mathbb{B}_{n}$ is the unit sphere and the triple $\left(A\left(\mathbb{B}_{n}\right), \overline{\mathbb{B}}_{n}, \mu\right)$ is regular for every measure $\mu \in M^{+}\left(\overline{\mathbb{B}}_{n}\right)$ with support contained in $S(A)$ (Proposition 2 in [1]). Hence every $A\left(\mathbb{B}_{n}\right)$-isometry is regular. By a result of Athavale [3] the $A\left(\mathbb{B}_{n}\right)$-isometries are precisely the spherical isometries, that is, the commuting tuples $T=\left(T_{1}, \ldots, T_{n}\right) \in L(\mathcal{H})^{n}$ satisfying the identity

$$
\sum_{i=1}^{n} T_{i}^{*} T_{i}=1_{\mathcal{H}}
$$

Thus in this case, Theorem 1 specializes to a recent result of Didas [10]. 
If $D=\mathbb{D}^{n}$ is the open unit polydisc, then $S(A)=\mathbb{T}^{n}$ is the $n$-torus and again each $A\left(\mathbb{D}^{n}\right)$-isometry is regular. The $A\left(\mathbb{D}^{n}\right)$-isometries are precisely the commuting $n$-tuples $T \in L(\mathcal{H})^{n}$ of isometries and Theorem 1 reduces to the cited result of $\mathrm{Li}$ and McCarthy [13] on the reflexivity of finite commuting families of isometries.

More generally, in 2] (Theorem 3) Aleksandrov described conditions which ensure that the triple $(A, K, \mu)$ is regular for every measure $\mu \in M^{+}(K)$ with support contained in the Shilov boundary $S(A)$ of $A$. Under these conditions every $A$ isometry is regular, and hence reflexive. It is well known (see [11) that these conditions of Aleksandrov hold for $A(D)$ when $D$ is a bounded symmetric and circled domain in $\mathbb{C}^{n}$ or when $D$ is a relatively compact strictly pseudoconvex open set in $\mathbb{C}^{n}$, or in a Stein submanifold $X$ of $\mathbb{C}^{n}$.

\section{REFERENCES}

[1] A.B. Aleksandrov, Inner functions on compact spaces, Funct. Anal. Appl. 18 (1984), 87-98. MR0745695 (86d:32003)

[2] A.B. Aleksandrov, Inner functions: Results, methods, problems, Proc. Int. Cong. Math., Berkeley, 1986, Vol. 1, 699-707. MR0934272 (89c:32015)

[3] A. Athavale, On the intertwining of joint isometries, J. Operator Theory 23 (1990), 339-350. MR.1066811 (91i:47029)

[4] H. Bercovici, A factorization theorem with applications to invariant subspaces and the reflexivity of isometries, Math. Res. Lett. 1 (1994), 511-518. MR.1302394 (95m:47005)

[5] H. Bercovici, C. Foias and C. Pearcy, Dual algebras with applications to invariant subspaces and dilation theory, CBMS Regional Conf. Ser. in Math. 56, Amer. Math. Soc., Providence, RI, 1985. MR0787041 (87g:47091)

[6] J.B. Conway, The theory of subnormal operators, Math. Surveys Monogr. 36, Amer. Math. Soc., Providence, RI, 1991. MR.1112128 (92h:47026)

[7] J.B. Conway, Towards a functional calculus of subnormal tuples: The minimal normal extension, Trans. Am. Math. Soc. 326 (1991), 543-567. MR1005077 (91k:47048)

[8] J.A. Deddens, Every isometry is reflexive, Proc. Amer. Math. Soc. 28 (1971), 509-512. MR0278099 (43:3831)

[9] M. Didas, Dual algebras generated by von Neumann $n$-tuples over strictly pseudoconvex sets, Dissertationes Math. 425 (2004). MR2067612 (2005d:47009)

[10] M. Didas, Spherical isometries are reflexive, Preprint, Univ. des Saarlandes, 2004.

[11] M. Didas and J. Eschmeier, Subnormal tuples on strictly pseudoconvex and bounded symmetric domains, Acta Sci. Math. (Szeged), to appear.

[12] D. Hadwin and E. Nordgren, Subalgebras of reflexive algebras, J. Operator Theory 7 (1982), 3-23. MR0650190 (83f:47033)

[13] W.S. Li and J. McCarthy, Reflexivity of isometries, Studia Math. 124 (1997) 101-105. MR:1447616 (98h:47009)

[14] V. Müller and M. Ptak, Spherical isometries are hyporeflexive, Rocky Mountain J. Math. 29 (1999), 677-683. MR.1705481 (2000i:47007)

[15] R. Olin and J. Thomson, Algebras of subnormal operators, J. Funct. Anal. 37 (1980), 271-301. MR0581424 (82a:47024)

[16] M. Putinar, Spectral inclusion for subnormal $n$-tuples, Proc. Amer. Math. Soc. 90 (1984), 405-406. MR0728357 (85f:47029)

[17] D. Sarason, Invariant subspaces and unstarred operator algebras, Pacific J. Math. 17 (1966), 511-517. MR0192365 (33:590)

Fachrichtung Mathematik, Universität des Saarlandes, Postfach 151150, D-66041 SaArbrücken, Germany

E-mail address: eschmei@math.uni-sb.de 